Acta Crystallographica Section D

Biological

Crystallography

ISSN 0907-4449

Antonello Merlino, ${ }^{\mathrm{a}, \mathrm{b}}$ Martin R. Fuchs, ${ }^{\mathrm{c}}$ Andrea Pica, ${ }^{\mathrm{a}}$ Anna

Balsamo, ${ }^{a}$ Florian S. N. Dworkowski, ${ }^{\mathrm{c}}$ Guillaume Pompidor, ${ }^{\mathrm{C}}$ Lelio Mazzarella ${ }^{\mathrm{a}}$ and Alessandro Vergara ${ }^{a, b *}$

${ }^{a}$ Department of Chemical Sciences, University of Naples 'Federico II', Via Cintia, I-80126 Naples, Italy, ${ }^{\mathbf{b}}$ Istituto di Biostrutture e Bioimmagini, CNR, Naples, Italy, and ${ }^{\mathbf{c} S w i s s}$ Light Source, Paul Scherrer Institute, Villigen, Switzerland

Correspondence e-mail: avergara@unina.it

Received 24 July 2012

Accepted 9 October 2012

\section{Selective X-ray-induced NO photodissociation in haemoglobin crystals: evidence from a Raman- assisted crystallographic study}

Despite their high physiological relevance, haemoglobin crystal structures with NO bound to haem constitute less than $1 \%$ of the total ligated haemoglobins (Hbs) deposited in the Protein Data Bank. The major difficulty in obtaining NOligated $\mathrm{Hbs}$ is most likely to be related to the oxidative denitrosylation caused by the high reactivity of the nitrosylated species with $\mathrm{O}_{2}$. Here, using Ramanassisted X-ray crystallography, it is shown that under X-ray exposure (at four different radiation doses) crystals of nitrosylated haemoglobin from Trematomus bernacchii undergo a transition, mainly in the $\beta$ chains, that generates a pentacoordinate species owing to photodissociation of the $\mathrm{Fe}-\mathrm{NO}$ bond. These data provide a physical explanation for the low number of nitrosylated $\mathrm{Hb}$ structures available in the literature.

\section{Introduction}

Nitrogen monoxide (NO) is a signalling molecule involved in the regulation of essential physiological processes (Moncada et al., 1991). Endogenous NO is produced by metabolism of $\mathrm{L}$-arginine catalyzed by the enzyme NO synthetase (with haem as a co-enzyme) and by the nitrite reductase activity of deoxy hemoglobin $(\mathrm{Hb})$. Thus, there is great interest in understanding the NO-haem interaction, which is of tremendous physiological importance and is vital to a number of biological processes, including neurotransmission, vasodilation and blood clotting. The interaction between $\mathrm{NO}$ and haemoproteins is relevant to all of these processes, with many iron-containing proteins being modulated in vivo by NO (Bonaventura et al., 2004; Chan et al., 1998). The molecular mechanisms of many of these interrelated biochemical pathways are still controversial (Moncada et al., 1991).

Reactivity of NO with Hbs has been shown both at the haem and the cysteine residues (Chan et al., 1998). However, NO and related reactive species also react with other amino-acid residues, such as Tyr (Martínez-Ruiz et al., 2011) and Trp (Suntsova et al., 2002). Moreover, NO-mediated chemical modification of amino-acid residues may affect the reactivity of the haem. Crystal structures of human $\mathrm{Hb}$ (HbA) $S$-nitrosylated at Cys93 $\beta$ (Chan et al., 1998) and with NO bound at the haem iron (Chan et al., 2004) are both available. The study of such nitrosylated species is complicated by the high reactivity of haem-NO complexes, which undergo rapid oxidative denitrosylation under aerobic conditions. The nitrosylated $\mathrm{Hb}$ structures available in the literature were obtained via NO soaking into deoxygenated HbA crystals (Chan et al., 2004). The crystal structure of the fully liganded T-state complex of $\mathrm{HbA}$ with $\mathrm{NO}$ revealed the striking and surprising result that the bond between the haem iron and the proximal histidine in the $\alpha$ subunits is ruptured (Chan et al., 2004). This finding is in good agreement with the resonance Raman (Szabo \& Barron, 1975) and infrared (Maxwell \& Caughey, 1976) spectra, which exhibit a mixture of pentacoordinated and hexacoordinated NO adducts. In contrast, in the $\beta$ subunits the iron-proximal histidine bond is intact. It is also known that visible laser-induced photodissociation can be a minor source of denitrosylation, but with very low quantum yields in the visible region (Opisov et al., 2007). 
Here, we present an additional experiment-generated source of denitrosylation owing to X-ray photolysis of crystals of nitrosylated $\mathrm{Hb}$ from the Antarctic fish Trematomus bernacchii ( $\mathrm{HbTb})$. Crystal structures of $\mathrm{HbTb}$ are available in several ferrous (Camardella et al., 1992; Mazzarella et al., 2006) and ferric forms (Vergara et al., 2007, 2009; Merlino et al., 2011) at different pH values. Indeed, this system represents a model for the study of the peculiarities of fish $\mathrm{Hb}$ [such as the Root effect (Verde et al., 2008) and haemichrome formation (Verde et al., 2008; Vitagliano et al., 2008; Merlino et al., 2011)].

$\mathrm{X}$-ray-induced radiation damage is a frequent phenomenon, especially when third-generation synchrotrons are used. Several examples of photo-damage to protein side chains have been reported (Garman, 2010; Murray et al., 2004). Raman microscopy prior to and after X-ray data collection can be a valuable tool for the detection of such artifacts. Indeed, several papers on Raman-assisted crystallographic studies have recently been published; these include, as examples, Br dissociation from DNA (McGeehan et al., 2007) and disulfide reduction (Carpentier et al., 2010). Very recently, X-rayinduced NO photodissociation at low temperature was reported for HbA in solution via XANES studies (Arcovito \& Della Longa, 2011). However, to the best of our knowledge this is the first Raman-assisted crystallographic evidence for X-ray-induced NO photodissociation at a third-generation synchrotron (the Swiss Light Source). Indeed, the Raman spectra of the X-ray-exposed single crystals showed NO photodissociation that electron-density maps revealed to be localized mainly in the $\beta$ subunits. Ultimately, our combined crystallographic and spectroscopic method has provided (i) an experimental protocol for the detection of X-ray-induced NO photodissociation in haemoprotein crystals via Raman microscopy and (ii) a method to obtain approximate quantitative information on the relative Raman crosssection of the bands corresponding to deoxygenated and nitrosylated Hbs. This latter result may be useful to quantitatively evaluate the degree of NO photodissociation in future studies and as a reference for computational studies on resonance Raman (RR) spectra.

\section{Methods}

\subsection{Crystallization of NO-bound haemoglobin}

Deoxygenated $\mathrm{HbTb}$ crystals at $\mathrm{pH} 6$ were produced as reported previously (Mazzarella et al., 2006). Nitrosylation was achieved just prior to X-ray data collection via NO soaking of deoxygenated crystals. NO was produced in situ by adding a degassed equimolar solution of sodium dithionite and sodium nitrite $(30 \mathrm{mM})$. The soaking time (15 min) was defined via off-line Raman microscopy (see below).

\subsection{Raman microscopy}

2.2.1. Off-line Raman microscopy. In order to define the experimental nitrosylation protocol, preliminary RR microscopy spectra were collected from HbTb deoxy crystals in the Department of Chemical Sciences at University of Naples 'Federico II' using an apparatus described elsewhere (Vergara, Merlino et al., 2008). The excitation line was $514 \mathrm{~nm}\left(\mathrm{Ar}^{+}\right.$laser), with a power of $2 \mathrm{~mW}$ at the sample.

2.2.2. Online Raman microscopy. Prior to and after each collection of X-ray diffraction data, RR spectra were collected on beamline X10SA of the Swiss Light Source (SLS) using an upgraded version of the microspectrophotometer previously reported (Owen et al., 2009). RR spectra were collected from a crystal that was maintained in the same orientation. The excitation wavelength was $405 \mathrm{~nm}$ (Omicron LDM405.400 diode laser) with a power at the sample of $5 \mathrm{~mW}$ in a
$50 \mu \mathrm{m}$ round focal spot. Successive RR spectra were collected to rule out the occurrence of significant photodissociation upon laser exposure. Indeed, no detectable difference was observed in such successive RR spectrum (data not shown), as reported in the literature (Opisov et al., 2007).

\subsection{X-ray data collection}

Diffraction data were initially collected in-house using a Rigaku MicroMax-007 HF X-ray generator equipped with a Saturn944 CCD detector and a copper rotating-anode source. Using this conventional source, the resolution limit was $2.0 \AA$ (data not shown). Diffraction data for fully (NO-HbTb) and partially (NO_x1-HbTb, NO_x2-HbTb and NO_x3-HbTb) nitrosylated $\mathrm{HbTb}$ crystals were collected on beamline X10SA of the SLS at four different X-ray doses. X-ray diffraction data for NO_x1-HbTb, NO_x2-HbTb and NO_x3-HbTb were collected from the same crystal. Results similar to those described for NO-HbTb were obtained using a different crystal (NO$\mathrm{HbTb}$-bis). Data-collection statistics are reported in Supplementary Table S1. ${ }^{1}$ All data sets were collected at $100 \mathrm{~K}$ using glycerol as a cryoprotectant and were processed with the $H K L-2000$ program suite (v.7.01; Otwinowski \& Minor, 1997). An analysis of the collected diffraction data showed that the NO-HbTb crystals present pseudomerohedral twinning. This finding is in line with the previous results on deoxy crystals of the same $\mathrm{Hb}$ (Mazzarella et al., 2006). The twin fractions, as determined by the algorithm implemented in the program SHELX (Sheldrick, 2008), were close to 0.5 in all cases. The refinements were performed with SHELX (Sheldrick, 2008). The structures refined to the $R$ factor and $R_{\text {free }}$ values reported in Suppementary Table S1. Further structural details will be reported elsewhere. The X-ray dose was evaluated using the software RADDOSE (Paithankar et al., 2009). Owing to the large number of frames and the long exposure time used for in-house experiments, the total X-ray dose was comparable with that of the synchrotron X-ray data collection. The structure of NO-HbTb has been deposited in the Protein Data Bank as entry 4g51.

\section{Results and discussion}

The in-house NO soaking of deoxygenated $\mathrm{HbTb}$ crystals at $\mathrm{pH} 6$ was monitored via Raman microscopy with excitation at $514 \mathrm{~nm}$ (Supplementary Fig. S1). The best conditions to obtain a fully nitrosylated form were $30 \mathrm{~m} M$ sodium dithionite, $20 \mathrm{~m} M$ sodium nitrite with a 10 min soaking time. Soaking experiments were highly reproducible when degassed solutions and a reducing environment were used. NO formation was monitored by following the position and the intensity of Raman bands, which change from values typical of the deoxy form $\left(v_{4}=1355\right.$ and $v_{2}=1375 \mathrm{~cm}^{-1}$; Kitagawa et al., 1978; Spiro \& Strekas, 1974) to those typical of the nitrosylated form $\left(v_{4}=1370, v_{2}=1505\right.$ and $v_{10}=1635 \mathrm{~cm}^{-1}$; Stong et al., 1980$)$. The fully ligated form was also analyzed at higher spectral resolution in order to evaluate the NO coordination (Supplementary Fig. S1). The $v_{10}$ value of $1635 \mathrm{~cm}^{-1}$ revealed the presence of hexacoordinated $\mathrm{HbTb}$. No detectable bands for the pentacoordinated nitrosylated species, the $v_{10}$ of which would be expected to be at $1640 \mathrm{~cm}^{-1}$ (Szabo \& Barron, 1975), were observed. This finding indicates differing behaviour between $\mathrm{HbA}$ and $\mathrm{HbTb}$. Interestingly, the absence of the pentacoordinated species seems to be a common feature of fish $\mathrm{Hbs}$,

\footnotetext{
${ }^{1}$ Supplementary material has been deposited in the IUCr electronic archive (Reference: DW5025). Services for accessing this material are described at the back of the journal.
} 

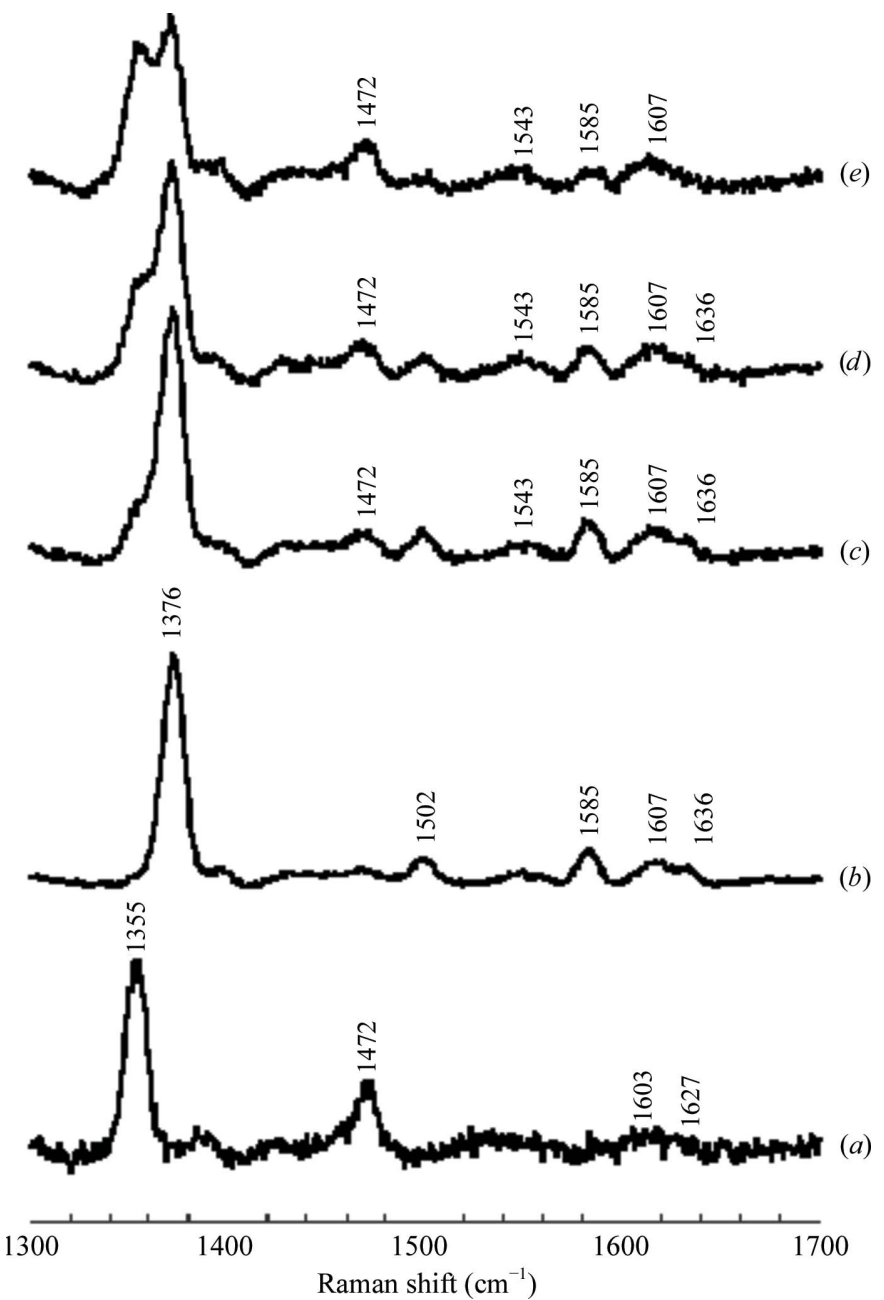

Figure 1

Resonance Raman spectra of $\mathrm{HbTb}$ crystals in the deoxygenated form $(a)$, the fully nitrosylated form (NO-HbTb) $(b)$ and the partially ligated forms $\mathrm{NO}_{-} \mathrm{x} 1-\mathrm{HbTb}(c)$, NO_x2-HbTb $(d)$ and $\mathrm{NO}_{-} \times 3$ - $\mathrm{HbTb}(e)$ arising from X-ray exposure at three different doses: 4.0, 7.6 and 11.2 MGy, respectively. The excitation line is $405 \mathrm{~nm}$ $(5 \mathrm{~mW}$ at the sample) and the exposure time is $300 \mathrm{~s}$. The spectral resolution is $8 \mathrm{~cm}^{-1}$.
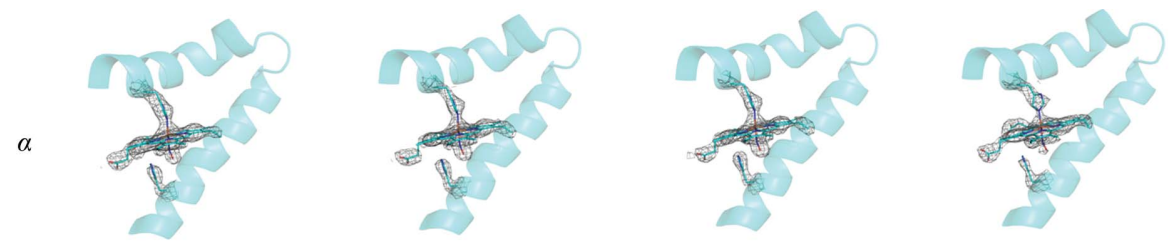

X-ray dose
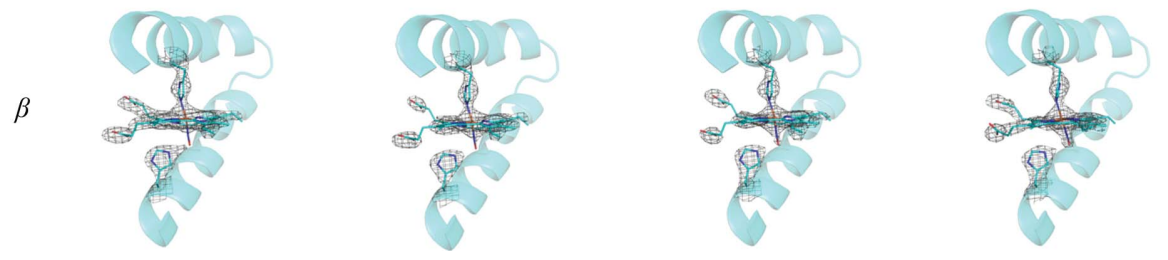

Figure 2

Difference Fourier electron-density maps $\left(2 F_{\mathrm{o}}-F_{\mathrm{c}}\right)$ of the $\alpha_{1}$ and $\beta_{1}$ haems of NO-HbTb and partially ligated forms (NO_x1-HbTb, NO_x2-HbTb and NO_x3-HbTb) at different X-ray doses $(0.4,4.0,7.6$ and $11.2 \mathrm{MGy}$, respectively). The maps were prepared using data at the same resolution $(2.5 \AA)$ and at the same electron-density level $(1.0 \sigma)$ since carp $\mathrm{Hb}$ also lacks the spectral characteristics typical of the pentacoordinated species (Scholler et al., 1979).

Despite the reproducibility of the soaking protocol, which was monitored via RR, several in-house X-ray diffraction data collections using a wavelength of $1.54 \AA$ only resulted in partial NO coordination limited to the $\alpha$ subunits (data not shown). Since oxidative denitrosylation was presumed, a protective environment (a mixture of ascorbate and catalase) was used. Even under these oxygen-free conditions, only partial binding was again obtained.

Simultaneous X-ray data collection assisted by online Raman spectroscopy prior to and after data collection at the SLS synchrotron (using a 1 A wavelength) solved the puzzle. Indeed, RR data (with excitation at $405 \mathrm{~nm}$ ) at the SLS prior to X-ray exposure showed complete binding of NO to haem prior to the diffraction experiment (Fig. 1), as revealed by $v_{4}$ and $v_{3}$ bands typical of hexacoordinated NO haemoglobin. The RR data collected at increasing X-ray doses revealed a detectable decrease in the low-spin (LS) signal and the appearance of a new high-spin (HS) signal. In particular, the X-ray exposure produced an almost $50 \%$ decrease in the NO band in favour of the deoxygenated form. Therefore, the RR data clearly showed an $\mathrm{X}$-ray-induced NO photodissociation. Moreover, no additional bands that could be associated with a transient tetracoordinate haem (with $\mathrm{NO}$ and both proximal and distal His not bound to iron), as disputed for HbA (Arcovito \& Della Longa, 2011), were observed in the HbTb RR spectra.

The spectroscopic data correlate well with the crystallographic evidence. No evidence of NO binding to Cys, Tyr and Trp residues was apparent (data not shown); therefore, we will focus on the haem pockets. $2 F_{\mathrm{o}}-F_{\mathrm{c}}$ electron-density maps of the $\alpha_{1}$ and $\beta_{1}$ chains of fully ligated $\mathrm{NO}-\mathrm{HbTb}$ refined at the moderate resolution of $2.5 \AA$ are shown in Fig. 2. A comparison of these maps and those calculated from the structures refined using data collected at increasing X-ray doses provides a clear interpretation of the changing intensity of the NO signal (Fig. 2). Indeed, it is evident that NO photodissociation occurs to different degrees in the $\alpha$ and $\beta$ subunits (Fig. 2). In particular, NO photodissociation is almost confined to the $\beta$ haems (Fig. 2). The electron density of the NO molecule is already very poor in the $\beta_{2}$ haem in fully ligated NO-HbTb and in the $\alpha_{2}$ haem of NO_x3-HbTb. In order to make the comparison between the Raman data and the crystallographic data as consistent as possible, the occupancy factors of NO were estimated in the different structures and are reported in Table 1 together with the X-ray dose used for the corresponding data collection and the ratio between the intensity of the $v_{4}$ or $v_{2}$ bands corresponding to the NO and deoxygenated species. This strong heterogeneity between the $\alpha$ and $\beta$ haems is a well known phenomenon both in ferric (Vergara, Vitagliano et al., 2008; Balsamo et al., 2012) and deoxy fish Hbs (Vergara et al., 2010).

NO photodissociation is probably a source of denitrosylation in all globins. Indeed, recent solution studies have revealed X-ray-induced photodissociation of $\mathrm{NO}$ in $\mathrm{HbA}$ at low temperature (Arcovito \& Della Longa, 2011). The only available NObound structure of $\mathrm{HbA}$ was collected at moderate resolution (at low X-ray dose) and at room temperature (Chan et al., 1998). Therefore, our Raman microscopic evidence of $\mathrm{NO}$ photodissociation for $\mathrm{Hb}$ from 
Table 1

Estimated occupancy factors and relative intensities of some Raman bands for the photodissociated and nitrosylated species.

\begin{tabular}{lclll}
\hline & $\begin{array}{l}\text { Total dose } \\
(\mathrm{MGy})\end{array}$ & $\begin{array}{l}\text { NO occupancy } \\
\left(\alpha_{1}, \beta_{1}, \alpha_{2}, \beta_{2}\right)\end{array}$ & $I_{1355} / I_{1370}$ & $I_{1475} / I_{1505}$ \\
\hline HbTb-NO & 0.4 & $1.0,1.0,1.0,0.7 \dagger$ & 0 & 0 \\
NO_x1-HbTb & 4.0 & $1.0,0.8,1.0,0.7$ & 0.2 & 1.2 \\
NO_x2-HbTb & 7.6 & $1.0,0.6,1.0,0.6$ & 0.6 & 1.3 \\
NO_x3-HbTb & 11.2 & $1.0,0.4,0.4,0.4$ & 0.8 & 2.8 \\
\hline
\end{tabular}

$\dagger$ The electron density of HbTb-NO is very poor in the $\beta_{2}$ chain.

T. bernacchii may have a general impact, since it may explain the surprisingly small number of crystal structures of nitrosyl Hbs deposited in the PDB. Indeed, while only a limited number of literature sources report the absence of the expected NO bound to the haemoprotein structure (Forouhar et al., 2007), an unpredictably large number of unpublished data could be retained in scientists' drawers as negative observations. The methodological take-home message of this work is the advice to monitor NO binding via Raman microscopy prior to and after X-ray diffraction data collection, while keeping the dose as low as possible. Finally, it is worth noting that data such as those reported in Table 1 (relative Raman intensities and occupancy factors) collected at the same crystal orientation can provide relative resonance Raman cross-sections of different coordination states. However, this evaluation does require that the orientation dependence of the modes belonging to the different coordination states is identical. It is worth mentioning that calculations of resonance Raman spectra are still considered to be a formidable task (Santoro et al., 2011) and this type of spectroscopic and structural data represent valuable input data for a current challenge in computational chemistry.

PNRA is acknowledged for financial support. SLS is acknowledged for travel grants to AM and AV. Umberto Oreste is acknowledged for providing T. bernacchii haemolysate.

\section{References}

Arcovito, A. \& Della Longa, S. (2011). Inorg. Chem. 50, 9423-9429.

Balsamo, A., Sannino, F., Merlino, A., Parrilli, E., Tutino, M. L., Mazzarella, L. \& Vergara, A. (2012). Biochimie, 94, 953-960.

Bonaventura, C., Fago, A., Henkens, R. \& Crumbliss, A. L. (2004). Antioxid. Redox Signal. 6, 979-991.

Camardella, L., Caruso, C., D'Avino, R., di Prisco, G., Rutigliano, B., Tamburrini, M., Fermi, G. \& Perutz, M. F. (1992). J. Mol. Biol. 224, 449-460. Carpentier, P., Royant, A., Weik, M. \& Bourgeois, D. (2010). Structure, 18, $1410-1419$
Chan, N.-L., Kavanaugh, J. S., Rogers, P. H. \& Arnone, A. (2004). Biochemistry, 43, 118-132.

Chan, N.-L., Rogers, P. H. \& Arnone, A. (1998). Biochemistry, 37, 16459 16464.

Forouhar, F. et al. (2007). Proc. Natl Acad. Sci. USA, 104, 473-478.

Garman, E. F. (2010). Acta Cryst. D66, 339-351.

Kitagawa, T., Abe, M. \& Ogoshi, H. (1978). J. Chem. Phys. 69, 4516-4525.

Martínez-Ruiz, A., Cadenas, S. \& Lamas, S. (2011). Free Radic. Biol. Med. 51, $17-29$.

Maxwell, J. C. \& Caughey, W. S. (1976). Biochemistry, 15, 388-396.

Mazzarella, L., Vergara, A., Vitagliano, L., Merlino, A., Bonomi, G., Scala, S., Verde, C. \& di Prisco, G. (2006). Proteins, 65, 490-498.

McGeehan, J. E., Carpentier, P., Royant, A., Bourgeois, D. \& Ravelli, R. B. G. (2007). J. Synchrotron Rad. 14, 99-108.

Merlino, A., Howes, B. D., Prisco, G., Verde, C., Smulevich, G., Mazzarella, L. \& Vergara, A. (2011). IUBMB Life, 63, 295-303.

Moncada, S., Palmer, R. M. \& Higgs, E. A. (1991). Pharmacol. Rev. 43, 109-142.

Murray, J. W., Garman, E. F. \& Ravelli, R. B. G. (2004). J. Appl. Cryst. 37, 513-522.

Opisov, A. N., Borisenko, G. G. \& Vòladimirov, Y. A. (2007). Biochemistry (Moscow), 44, 259-292.

Otwinowski, Z. \& Minor, W. (1997). Methods Enzymol. 276, 307-326.

Owen, R. L., Pearson, A. R., Meents, A., Boehler, P., Thominet, V. \& SchulzeBriese, C. (2009). J. Synchrotron Rad. 16, 173-182.

Paithankar, K. S., Owen, R. L. \& Garman, E. F. (2009). J. Synchrotron Rad. 16, $152-162$.

Santoro, F., Cappelli, C. \& Barone, V. (2011). J. Chem. Theory Comput. 7, 1824-1829.

Scholler, D. M., Wang, M.-Y. R. \& Hoffman, B. M. (1979). J. Biol. Chem. 254, 4072-4078.

Sheldrick, G. M. (2008). Acta Cryst. A64, 112-122.

Spiro, T. G. \& Strekas, T. C. (1974). J. Am. Chem. Soc. 96, 338-345.

Stong, J. D., Spiro, T. G., Kubaska, R. J. \& Shupack, S. I. (1980). J. Raman Spectrosc. 9, 312-314.

Suntsova, T. P., Beda, N. V. \& Nedospasov, A. A. (2002). IUBMB Life, 54, 281-292.

Szabo, A. \& Barron, L. D. (1975). J. Am. Chem. Soc. 97, 660-662.

Verde, C., Vergara, A., Mazzarella, L. \& di Prisco, G. (2008). Curr. Protein Pept. Sci. 9, 578-590.

Vergara, A., Franzese, M., Merlino, A., Bonomi, G., Verde, C., Giordano, D., di Prisco, G., Lee, H. C., Peisach, J. \& Mazzarella, L. (2009). Biophys. J. 97, 866-874.

Vergara, A., Franzese, M., Merlino, A., Vitagliano, L., Verde, C., di Prisco, G., Lee, H. C., Peisach, J. \& Mazzarella, L. (2007). Biophys. J. 93, 28222829

Vergara, A., Merlino, A., Pizzo, E., D’Alessio, G. \& Mazzarella, L. (2008). Acta Cryst. D64, 167-171.

Vergara, A., Vitagliano, L., Merlino, A., Sica, F., Marino, K., Verde, C., di Prisco, G. \& Mazzarella, L. (2010). J. Biol. Chem. 285, 32568-32575.

Vergara, A., Vitagliano, L., Verde, C., di Prisco, G. \& Mazzarella, L. (2008). Methods Enzymol. 436, 425-444.

Vitagliano, L., Vergara, A., Bonomi, G., Merlino, A., Verde, C., di Prisco, G., Howes, B. D., Smulevich, G. \& Mazzarella, L. (2008). J. Am. Chem. Soc. 130, 10527-10535. 